\title{
HPV: a importância da vacinação para redução do surgimento de lesões pré-malignas do câncer de colo uterino
}

\author{
HPV: the importance of vaccination to reduce the onset of pre-malignant lesions of cervical \\ cancer
}
HPV: la importancia de la vacunación para reducir la aparición de lesiones premalignas del cáncer de cuello uterino

Ana Júlia Vilela Faria ${ }^{1 *}$, Ana Clara Fernandes Barroso ${ }^{2}$, Ana Paula Soares Lacerda ${ }^{3}$, Bruna Marçal Carvalho Mendes ${ }^{4}$, Camila Euqueres Partata ${ }^{4}$, Caroline Lopes de Araújo ${ }^{5}$, Francine Correia dos Santos ${ }^{6}$, Maria Eduarda Melo Alves Freitas ${ }^{7}$, Maria Vitória Almeida Moreira ${ }^{8}$, Anna Carla Garcia Cabral ${ }^{9}$.

\begin{abstract}
RESUMO
Objetivo: Revisar a literatura destacando a importância e os principais benefícios da vacinação para a redução de lesões pré-malignas do câncer de colo uterino. Revisão bibliográfica: O Papilomavírus Humano (HPV) causa infecções que são propulsoras de lesões intraepiteliais e que podem levar ao câncer de colo uterino, sendo a neoplasia com maior incidência e mortalidade no sexo feminino. A infecção se dá por meio do contato sexual e o Papanicolau é o principal exame diagnóstico, detectando lesões pré-cancerígenas. A principal profilaxia contra o HPV é a vacinação, que demonstrou considerável redução de lesões precursoras na população alvo dos países que adotaram a vacina, evidenciando sua eficácia. A sensação de proteção proporcionada pela cobertura vacinal no decorrer dos anos, contudo, resultou em descaso e certa resistência à vacinação. Somado a isso, o desconhecimento das jovens e da família quanto à segurança, ao benefício e aos efeitos adversos da vacina atuam como entraves para a plena adesão à vacinação no país. Considerações finais: Visa a importância e os resultados da profilaxia vacinal na ginecologia e obstetrícia, tornando-se imprescindível o entendimento da relevância social dessa imunização como questão de saúde pública.
\end{abstract}

Palavras-chave: HPV, Vacinação, Câncer de colo uterino.

\begin{abstract}
Objective: Review the literature highlighting the importance and main benefits of vaccination for the reduction of premalignant lesions of cervical cancer. Bibliographic review: Human Papillomavirus (HPV) causes infections that are propellants of intra-epithelial lesions and that can lead to cervical cancer, being the neoplasia with the highest incidence and mortality in females. The infection occurs through sexual contact and Pap smear is the main diagnostic test, detecting precancerous lesions. The main prophylaxis against HPV is vaccination, which has shown a considerable reduction in precursor lesions in the target population of countries that have adopted the vaccine, demonstrating its effectiveness. The feeling of protection provided by vaccination coverage over the years, however, has resulted in neglect and a certain resistance to vaccination. In addition, the lack of knowledge of young women and their families about the safety, benefits and adverse effects of the vaccine act as barriers to full adherence to vaccination in the country. Final considerations: It aims at the importance and results of vaccine prophylaxis in gynecology and obstetrics, making it essential to understand the social relevance of this immunization as a public health issue.
\end{abstract}

Keywords: HPV, Vaccination, Uterine cervical neoplasms.

1 Universidade Nove de Julho (UNINOVE), Guarulhos - SP. *E-mail: anaclarafbarroso1@gmail.com

2 Universidade de Gurupi (UNIRG), Gurupi - TO.

${ }^{3}$ Centro Universitário de Belo Horizonte (UniBH), Belo Horizonte - MG.

${ }^{4}$ Centro Universitário Imepac Araguari (IMEPAC), Araguari - MG.

${ }_{5}^{5}$ Unifacisa Centro Universitário (UNIFACISA), Campina Grande - PB.

6 Universidade Nova Iguaçu (UNIG), Nova Iguaçu - RJ.

7 Universidade Federal de Lavras (UFLA), Lavras - MG.

8. Universidade Nilton Lins (UNL), Manaus - AM.

9 Universidade Católica de Brasília (UCB), Brasília - DF. 


\section{RESUMEN}

Objetivo: Revisar la literatura, destacando la importancia y los principales beneficios de la vacunación orientada a la reducción de lesiones pre malignas de cáncer de cuello uterino. Revisión bibliográfica: El papilomavirus humano (VPH) provoca infecciones que son propulsoras de lesiones intraepiteliales y que pueden derivar en cáncer de cuello uterino, siendo la neoplasia de mayor incidencia y mortalidad en el sexo femenino. La infección se produce por contacto sexual y la prueba de Papanicolaou es la principal prueba diagnóstica, detectando lesiones precancerosas. La principal profilaxis contra el VPH es la vacunación, que ha mostrado una considerable reducción de las lesiones precursoras en la población diana de los países que han adoptado la vacuna, demostrando su efectividad. Sin embargo, la sensación de protección proporcionada por la cobertura de vacunación a lo largo de los años ha resultado en el abandono y una cierta resistencia a la vacunación. Además, el desconocimiento de las mujeres jóvenes y sus familias sobre la seguridad, los beneficios y los efectos adversos de la vacuna actúan como barreras para la plena adhesión a la vacunación en el país. Consideraciones finales: Apunta a la importancia y resultados de la profilaxis vacunal en ginecología y obstetricia, por lo que es fundamental comprender la relevancia social de esta inmunización como un tema de salud pública.

Palabras clave: HPV, Vacunación, Neoplasias del cuello uterino.

\section{INTRODUÇÃO}

As infecções causadas pelo Papilomavírus Humano (HPV) são importantes precursoras de lesões intraepiteliais do trato genital e oral, podendo se desenvolver em Câncer de Colo do Útero (CCU) e diversos outros tipos de tumores malignos, como o peniano, o vaginal, o anal e o orofaríngeo. Dentre essas condições, o CCU mostra-se a neoplasia com maior incidência e mortalidade entre pessoas do sexo feminino, apesar de já existirem vacinas eficazes como medida profilática às doenças decorrentes do HPV (RIBEIRO DV, et al., 2020; TOMASI E, et al., 2015)

Segundo Azevedo DS, et al. (2018), o Papilomavírus Humano constitui uma família com mais de 100 subtipos, dos quais cerca de 20 conseguem infectar o DNA das células epiteliais basais e provocar lesões pré-cancerígenas na pele e nas mucosas do trato aero-digestivo superior ou anogenital. A contaminação se dá por meio do contato sexual e estima-se que uma em cada dez mulheres é exposta já no começo de sua vida sexual, sendo que $46 \%$ delas já terão sido contaminadas após 3 anos (CARDIAL MFT, et al., 2019).

A diminuição dos índices de mortalidade e incidência da doença variam de acordo com a elevada adesão ao papanicolau (em torno de $80 \%$ das mulheres em idade de risco devem realizar o exame regularmente), a qualidade dos procedimentos de coleta (rapidez dos resultados) e o tratamento adequado. O Papanicolau é o principal exame preventivo e diagnóstico de HPV, além de ser economicamente viável e mostrar-se altamente específico, pode reduzir tratamentos e intervenções desnecessárias, o que faz com que seja um diagnóstico preventivo muito eficaz, pois detecta as lesões pré-cancerígenas em suas fases iniciais (TOMASI E, et al., 2015).

A Organização Mundial da Saúde (OMS) orienta a vacinação contra o HPV principalmente para meninas entre 9 e 14 anos, uma vez que a vacina tem sua eficácia máxima antes da iniciação sexual e consequentemente antes delas serem expostas à infecção. Estudos também mostram que a proteção da vacina se estende mesmo para aquelas mulheres que já iniciaram a vida sexual ou que receberam tratamento de lesões pelo Papilomavírus Humano, pois em última análise a vacina será uma proteção adicional ao sistema imunológico já comprometido. Além disso, elevadas taxas de vacinação para meninas reduzem também o risco de infecção por HPV em meninos (CARVALHO AMC, et al., 2019; CARDIAL MFT, et al., 2019).

A proteção proporcionada pelo sucesso dos programas de vacinação ao longo das décadas possibilitou uma falsa sensação de segurança contra as consequências fatais das doenças que são prevenidas pelas vacinas, ou seja, os males impedidos pela imunização em massa passaram a ser subestimados. Essa 
situação paradoxal dá origem à hesitação vacinal, que induz uma espécie de debate entre a liberdade de escolha do indivíduo e sua responsabilidade social na prevenção e propagação de doenças no meio em que vive (FONSECA VIEGAS SM, et al., 2019).

De acordo com Sabeena $S$, et al. (2018), visões sociais e mitos sobre sexo em uma sociedade conservadora podem mascarar os riscos reais à saúde associados ao vírus. Esses estigmas religiosos e moralistas, somados à necessidade da vacinação já em crianças e pré-adolescentes, associam de forma equivocada a vacinação contra o HPV, geralmente uma Infecção Sexualmente Transmissível (IST), à um comportamento sexual precoce. Dessa forma, tal pensamento contribui para um desinteresse por parte dos pais na vacinação de seus filhos (SIPP D, et al., 2018).

São necessárias intervenções governamentais que tratem a vacinação do HPV como uma questão de saúde pública e não um combate restrito à uma IST, desmistificando o vínculo da vacina com a iniciação sexual de crianças e adolescentes, promovendo a imunização de uma doença tão grave que precede vários tipos de câncer. Devido à baixa cobertura, a incidência do câncer do colo do útero se mantém alta no Brasil há vários anos, apesar de inúmeros programas públicos e incentivos à vacinação (PASSOS MRL e JUNIOR JE, 2019; FEDRIZZI EN, 2019).

Segundo Gomes JM, et al. (2020), para assegurar resultados consistentes e diminuir a frequência de lesões pré-cancerígenas, o alcance da vacinação completa necessita superar $80 \%$ do grupo prioritário. Conforme o planejamento de imunização brasileiro, esse público representa jovens do sexo feminino, com idade entre 9 a 14 anos e do sexo masculino, de 11 a 14 anos. Entretanto, dados mostram que a adesão à vacina contra o HPV (duas doses) em adolescentes do sexo feminino foi de $44,6 \%$, no Brasil. Vale ressaltar que em países como a Austrália, que também promovem a vacinação em ambiente escolar, a cobertura é mais alta (alcançando os mais de $80 \%$ ), do que aqueles em que ela só acontece nas instituições de saúde (MONTEIRO DLM, et al., 2015).

É recomendável que as vacinas contra o HPV façam parte do Plano Nacional de Imunização (PNI) dos países que relatam alta incidência de câncer relacionados ao Papilomavírus Humano. Ao implementar a vacinação, garantir a neutralidade de gênero e educar os pais acerca das formas não genitais de câncer associados ao HPV e os modos não sexuais de transmissão, são sugestões que visam maior adesão na imunização de seus filhos, desconstruindo os estigmas atrelados à vacina. Outrossim, é importante que informações claras sobre a vacina do HPV sejam amplamente divulgadas, a fim de descartar todas as informações falsas que a prejudicam e, enfim, combater a desinformação (SABEENA S, et al., 2018; SIPP D, et al., 2018).

Assim, essa revisão bibliográfica teve como objetivo enfatizar a importância da vacinação contra o Papilomavírus Humano na prevenção do surgimento de lesões pré-malignas e a relevância social dessa imunização como questão de saúde pública.

\section{REVISÃO BIBLIOGRÁFICA}

O câncer de colo do útero contribui com a morbimortalidade entre as mulheres na América Latina, no Caribe e no Brasil, sendo fundamental a prevenção, a detecção precoce e a promoção da saúde. Dessa forma, a identificação de genótipos virais e de fatores não virais do câncer de colo do útero, além do estímulo à pesquisa para verificar as especificidades em cada território, a detecção precoce das lesões precursoras e a realização de ações em promoção de saúde, como educação continuada para profissionais e educação em saúde no autocuidado da população em geral e das vulneráveis, são fundamentais para prevenção do HPV (SORPRESO ICE e KELLY PJ, 2018).

A prevenção primária da infecção pelo HPV pode ser feita pelo estímulo ao uso de preservativos e pela vacinação contra o vírus (MONTEIRO DLM, 2015). Atualmente existem três vacinas contra o HPV disponíveis: a tetravalente, a bivalente e a nonavalente. A vacina tetravalente protege contra os genótipos do HPV 16, 18, 6 e 11, tendo sido aprovada em 2006. A vacina bivalente protege contra os genótipos 16 e 18, sendo aprovada em 2007. E a nonavalente protege contra os genótipos 16, 18, 45, 31, 33, 52, 58, 6 e 1, e foi aprovada recentemente, nos EUA em 2014 e na Europa em 2015 (RIBEIRO JP e BORGES I, 2016). 
Segundo Ribeiro JP e Borges I (2016), a grande maioria dos casos de CCU apresentam infecção pelo HPV, sendo que 90\% são causados pelos genótipos de alto risco oncológico, onde os subtipos 16 e 18 mostram-se responsáveis por $70 \%$ dos casos, demonstrando uma maior prevalência. Por outro lado, os subtipos 31 e 45 provocam 10\% dos casos e o restante é consequência dos demais genótipos de alto grau. Em vista disso, é possível constatar que a aprovação da vacina nonavalente poderá promover maiores benefícios na imunização.

Vale a pena reforçar que, no Brasil, o Ministério da Saúde disponibiliza a vacina tetravalente para meninas de 9 a 13 anos, gratuitamente, no esquema de três doses $(0,5 \mathrm{~mL}-0,6$ e 60 meses) e para meninas e mulheres HIV-positivo entre 9 e 26 anos, com o esquema de três doses $(0,5 \mathrm{~mL}-0,1$ e 6 meses). As duas vacinas licenciadas no país (tetravalente e bivalente) estão disponíveis nas clínicas privadas de vacinação. Ambas as vacinas foram originalmente licenciadas utilizando esquema de três doses.

Com base em estudos, a Organização Mundial de Saúde (OMS) recomenda o esquema de duas doses da vacina tetravalente para meninas de 9 a 13 anos e, a vacina bivalente para meninas de 9 a 14 anos. (MONTEIRO DLM, 2015). Além disso, o Brasil disponibiliza as vacinas também para os meninos na faixa etária entre 11 e 14 anos. O país foi o primeiro da América do Sul e o sétimo no mundo a incluí-los no programa vacinal de vacinação contra o HPV (CARVALHO AMC, et al., 2019).

Ainda, vale enfatizar que, os países que adotaram a vacina relatam redução de lesões precursoras com variação de $60-80 \%$ e índices de $100 \%$ nas populações vacinadas com a quadrivalente $(6,11,16$ e 18$)$ para os casos de verrugas genitais (SORPRESO ICE e KELLY PJ, 2018). Além disso, estudos apontam que, a vacina bivalente ocasiona uma reação em que os títulos de anticorpos são maiores que a infecção em até 11 vezes e demonstram a manutenção da imunogenicidade e dos níveis de anticorpos por mais de 10 anos com ausência de falhas vacinais registradas (CARDIAL MFT, et al., 2017).

Ademais, ainda não se sabe por quanto tempo a vacina oferece total proteção às mulheres após o esquema vacinal completo, no entanto, é esperado que as vacinas forneçam proteção de até 20 anos. Até o momento, ainda não foi demonstrado necessidade de dose de reforço, entretanto, somente a continuação dos estudos indicará a necessidade ou não de mais doses (CARDIAL MFT, et al., 2017).

No Brasil, 9 a 10 milhões de pessoas encontram-se infectadas pelo HPV, sendo evidenciados 8.000 novos casos a cada ano, apresentando, pois, uma infecção epidêmica. Perante a isso, recentemente foi realizado um estudo composto por mulheres e homens, entre 16 e 25 anos, de todas as capitais do país e do Distrito Federal. Como resultado, $54,6 \%$ dos participantes apresentaram infecção pelo HPV, em que $38,4 \%$ eram por algum subtipo de alto risco (FEDRIZZI EN, 2019).

Nota-se que a grande maioria das infecções causadas pelo HPV é transitória, ocorrendo uma solução espontânea após dois anos em $90 \%$ dos casos, sem evidência de imunização contra o vírus. Dessa forma, mostra-se necessário a vacinação como forma de prevenir a infecção pelo HPV e, consequentemente, o desenvolvimento do Câncer de Colo de Útero (CCU). Vale ressaltar, que as vacinas contra o papilomavírus humano possuem caráter profilático, não sendo, portanto, capazes de eliminar a infecção (RIBEIRO JP e BORGES I, 2016).

De acordo com Cardial MF, et al. (2017), uma recente revisão sobre o impacto da vacina contra o HPV, constatou uma redução de aproximadamente $90 \%$ nas taxas de infecções pelos subtipos $6,11,16$ e 18 do HPV, e na ocorrência de verrugas genitais. Houve também diminuição de $45 \%$ e de $85 \%$ das anormalidades citológicas cervicais de baixo e de alto grau, respectivamente. Segundo Ribeiro DV, et al. (2020), foi realizado um estudo na República Checa que investigou a prevalência de lesões genitais verrucosas em um grupo de mulheres entre 16 e 40 anos. Como resultado, houve também redução de 90,6\% das lesões nas mulheres vacinadas há pelo menos um ano, demonstrando uma eficácia vacinal de $89 \%$.

Um outro grande estudo, realizado na Austrália em 2014, comparou a prevalência de lesões cervicais de alto grau entre mulheres vacinadas e não vacinadas contra HPV. Foi evidenciada uma eficácia de $46 \%$ na prevenção de lesões de alto grau e de $34 \%$ para outras anormalidades cervicais nas mulheres vacinadas, demonstrando uma significativa associação entre a vacina e a redução das lesões (RIBEIRO DV, et al., 2020). 
Sabe-se que a infecção viral se faz principalmente por meio do contato sexual, em que uma em cada dez mulheres se infectam no primeiro contato sexual. Perante a isso, é importante que a aplicação da vacina ocorra antes do início da atividade sexual para que sua eficácia seja máxima (CARDIAL MF, et al., 2017). Em mulheres sem infecção prévia, as vacinas promovem uma eficácia superior a 95\% contra lesões causadas pelos subtipos 16 e 18 do HPV (MONTEIRO DLM, et al., 2015).

Não obstante, um estudo realizado com mulheres na faixa etária de 15 a 26 anos, com histórico de infecção pelo HPV e realizando o tratamento, demonstrou que aquelas que receberam a vacina obtiveram redução de $64,9 \%$ a $86,3 \%$ no surgimento de novas lesões (CARDIAL MF, et al., 2017). Fedrizzi EN (2019), mencionou que recentes estudos mostraram benefícios da vacinação em mulheres já infectadas e até mesmo com neoplasia cervical intraepitelial estabelecida, apresentando uma redução de $75 \%$ a $88 \%$ na recorrência de lesões. Dessa forma, evidencia-se a importância de vacinar também as mulheres que já apresentam uma vida sexualmente ativa ou que já se infectaram.

Além das vacinas bivalente e tetravalente, existe ainda a vacina nonavalente (HPV9), que foi aprovada no Brasil pela Agência Nacional de Vigilância Sanitária (ANVISA) em dezembro de 2017, entretanto ainda se encontra indisponível no mercado nacional. Segundo Fedrizzi EN (2019), a vacina HPV9 apresenta uma proteção de $62,3 \%$ contra lesões cervicais de baixo grau (LSIL) e de $99,6 \%$ contra lesões de alto grau (HSIL). Dessa forma, pode-se concluir que a vacina nonavalente, é tão efetiva quanto as vacinas bivalente e tetravalente, além de oferecer uma proteção ampliada para cinco novos subtipos do HPV, apresentando potencial de prevenir $20 \%$ do CCU associado a esses subtipos (LOGROÑO IE, et al., 2018).

Os resultados da vacina para proteção contra HPV demonstram que há grande eficácia nas mulheres até os 25 anos, enquanto que nas idades mais avançadas ainda existem dúvidas sobre sua total eficiência. Contudo, a redução no risco de adquirir infecção persistente pelo HPV e/ou lesões precursoras de CCU, é comprovada após a administração das vacinas em mulheres entre 24 e 45 anos (RIBEIRO JP e BORGES I, 2016).

Apesar da existência de estudos que comprovem a eficácia das vacinas contra o HPV, ainda existem diversos aspectos culturais e sociais que dificultam a adesão ao esquema vacinal, prejudicando o maior alcance de cobertura vacinal no Brasil. Em um estudo, Faisal-Cury A, et al. (2020), demonstrou que apenas $48,9 \%$ das meninas brasileiras são vacinadas contra o HPV, sendo $83,5 \%$ entre as meninas que são alvo da estratégia pública de vacinação e apenas $21,8 \%$ entre as não elegíveis. Dessa forma, mostra-se essencial compreender os fatores que são empecilhos na adesão vacinal, para assim, instituir estratégias que incentivam um maior engajamento na vacinação.

Um estudo realizado por Viegas SMF, et al. (2019), com 605 adolescentes, para analisar o conhecimento do grupo sobre a vacinação, evidenciou inúmeras dúvidas acerca das vacinas em si, e das doenças imunopreveníveis como um todo. Questionaram a necessidade das vacinas, sua segurança e efeitos adversos. Esses dados refletem a falta de conhecimento dessa faixa etária sobre os riscos de doenças transmissíveis e sobre a importância de medidas profiláticas no combate a essas infecções.

Piqueras MC, et al. (2019), apontaram que os adolescentes consideravam a escola como o principal local de informação sobre promoção e educação em saúde. Ferrer $\mathrm{BH}$, et al., (2016), também relataram que a vacinação em ambiente escolar se apresentava como forte fator de adesão à vacinação por parte dos adolescentes. Essas conclusões evidenciam o grande benefício da utilização do ambiente escolar pelos profissionais de saúde em uma tentativa de obter atenção e engajamento dos estudantes acerca de temas de saúde individual e coletiva, bem como espaço para vacinação.

Contudo, um fator elencado que contribui para uma baixa adesão dessa faixa etária é a baixa adequação dos serviços de saúde às necessidades e linguagens dos jovens, que prejudica o acolhimento e os deixa pouco à vontade em conversar sobre assuntos mais íntimos, como sexo e infecções sexualmente transmissíveis, gerando uma comunicação ineficaz (VIEGAS SMF, et al., 2019).

Alder S, et al. (2015) e Alberts CJ, et al. (2017) relatam, por sua vez, que um importante fator de adesão, principalmente em pré-adolescentes, seria a devida informação dos pais em relação à vacina do HPV, 
sobretudo sua segurança, eficácia e ausência de efeitos adversos graves, com destaque especial para as mães. Ainda, foi constatado que um nível maior de conhecimento e escolaridade materna refletem em maiores taxas de imunização das filhas. O consentimento, o apoio e o incentivo familiar, portanto, mostram-se de extrema importância para o sucesso das campanhas vacinais. A existência de diálogo sobre sexualidade entre pais e filhos também parece estar associada a uma taxa maior de cobertura (FAISAL-CURY A, et al., 2020).

Ainda, como fator prejudicial, vale destacar o fácil acesso atualmente a conteúdos inverídicos na internet, o que pode contribuir para a perpetuação de opiniões desfavoráveis dos pais e para o afastamento dos adolescentes dos serviços de saúde. Mostra-se, portanto, a importância da presença dos órgãos de saúde nas redes, veiculando informações sobre a segurança e eficácia das vacinas, bem como expondo riscos das ISTs e contestando fake news (INTERAMINENSE INCS, et al., 2016).

Apesar dos empecilhos levantados em relação à população elegível, isto é, meninas entre 9 e 14 anos, outra barreira se mostra de grande relevância: a baixa cobertura da população não elegível para o programa público de vacinação contra o HPV. Dados da Pesquisa Nacional de Saúde do Escolar do Brasil de 2015 mostraram que, apesar da cobertura vacinal de mais de $80 \%$ da população elegível, apenas cerca de $20 \%$ das meninas não qualificadas buscavam a vacina em instituições privadas (FAISAL-CURY A, et al., 2020).

\section{CONSIDERAÇÕES FINAIS}

Diante destas observações, temos a relevância desse estudo ao relacionar a queda da prevalência do câncer de colo de útero no público feminino vacinado. Com isso, é digno de nota a importância da vacinação para prevenção dessa doença. Em síntese, a dificuldade de implementação da vacina quadrivalente contra o vírus HPV no calendário vacinal das adolescentes do sexo feminino no país se dá devido a existência de aspectos como, o acesso à conteúdos inverídicos na internet que distorcem o benefício que a vacina representa e também a necessidade de aprimoramento e melhor qualificação dos profissionais de saúde neste assunto, Destarte, faz-se necessário realizar mudanças na Atenção Básica, com o intuito de ampliar a visão da sociedade, quanto a importância da vacinação contra o Papilomavírus Humano e sua forma de prevenção contra o surgimento de lesões pré-malignas do câncer de colo uterino e a relevância social desta imunização como questão de saúde pública.

\section{REFERÊNCIAS}

1. ALBERTS CJ, et al. A longitudinal study on determinants of HPV vaccination uptake in parents/guardians from different ethnic backgrounds in Amsterdam, the Netherlands. BMC Public Health, 2017; 17(1): 220.

2. ALDER S, et al. Mothers' acceptance of human papillomavirus (HPV) vaccination for daughters in a country with a high prevalence of HPV. Oncol Rep, 2015; 33(5): 2521-8.

3. AZEVEDO DS, et al. Analysis of database about vaccination against Human Papillomavirus in Sergipe in 2014. DST j. bras. doenças sex. transm, 2018; 30 (2): 55-60.

4. CARDIAL MFT, et al. Papilomavírus humano (HPV). In: Programa vacinal para mulheres. FEMININA, 2019; 47(2): 94100.

5. CARVALHO AMC, et al. Adesão à vacina HPV entre os adolescentes: revisão integrativa. Texto Contexto Enferm, 2019; 28: e20180257.

6. FONSECA VIEGAS SM, et al. Preciso mesmo tomar vacina? Informação e conhecimento de adolescentes sobre as vacinas. Av Enferm, 2019, 37(2): 217-226.

7. CARVALHO AMC, et al. Adesão à vacina HPV entre os adolescentes: revisão integrativa. Texto Contexto Enferm, 2019; 28 (20180257).

8. FAISAL-CURY A, et al. Taxas de cobertura vacinal e preditores de vacinação contra HPV entre adolescentes elegíveis e não elegíveis no programa público de vacinação contra HPV brasileiro. BMC Public Health, 2020; $20: 458$.

9. FEDRIZZI EN. Why is the nonavalent HPV vaccine so important for Brazil? DST- J bras Doenças Sex Transm, 2019 ; $31(2): 39-42$.

10. FERRER BH, et al. Barriers and facilitators to uptake of the school- based HPV vaccination programme in an ethnically diverse group of young women. J Public Health (Oxf), 2016; 38(3): 569-77.

11. GOMES JM, et al. Human Papillomavirus (HPV) and the quadrivalent HPV vaccine among Brazilian adolescents and parents: Factors associated with and divergences in knowledge and acceptance. Plos One, 2020; $15(11)$ : 0241674.

12. INTERAMINENSE INCS, et al. Educational technologies to promote vaccination against human papillomavirus: integrative literature review. Texto Contexto Enferm, 2016; 25(2): e2300015. 
13. LOGROÑO IEN, et al. Eficacia de la vacuna nonavalente en la prevención de la infección por Virus Papiloma Humano (HPV) y cáncer cervical. CSSN, 2018; 9(2): 30-37.

14. MONTEIRO DLM, et al. Transformando vacina em vacinação: a importância da recomendação médica. Femina, 2015; 43(5): 193-196.

15. PASSOS MRL, JUNIOR JE. Editors' note on nonavalent HPV vaccine (9HPV). DST- J bras Doenças Sex Transm, 2019; 31(2): 43-44

16. PIQUERAS MC, et al. Reticencia vacunal: análisis del discurso de madres y padres con rechazo total o parcial a las vacunas. Gaceta Sanit, 2019; 33(1): 53-9.

17. RIBEIRO DV, et al. The impact of the HPV vaccine on the world: initial outcomes and challenges. DST- J bras Doenças Sex Transm, 2020; 32: 1-7.

18. RIBEIRO JP, BORGES I. Efficacy of the Vaccines Against Human Papillomavirus in Women Older than 24 Years in the Cervix Cancer Prevention. Acta Médica Portuguesa, 2016; 29 (6): 401-408.

19. SABEENA S, et al. Global human papilloma virus vaccine implementation: An update. The journal of Obstetrics and Gynaecology Research, 2018; 44(6): 989-997.

20. SIPP D, et al. No Vacillation on HPV Vaccination. Cell, 2018; 172(6): 1163-1167.

21. TOMASI E, et al. Estrutura e processo de trabalho na prevenção do câncer de colo de útero na Atenção Básica à Saúde no Brasil: Programa de Melhoria do Acesso e da Qualidade - PMAQ. Rev. Bras. Saúde Matern. Infant., 2015; 15(2): 171-180. 